\title{
Anti-PD-1 Monoclonal Antibody BAT1306
}

National Cancer Institute

\section{Source}

National Cancer Institute. Anti-PD-1 Monoclonal Antibody BAT1306. NCI Thesaurus.

Code C162871.

A recombinant, humanized monoclonal antibody directed against the negative immunoregulatory human cell receptor programmed cell death protein 1 (PD-1; PDCD1; CD279), with potential immune checkpoint inhibitory and antineoplastic activities. Upon administration, anti-PD-1 monoclonal antibody BAT 1306 targets, binds to and inhibits PD-1 and its downstream signaling pathways. This may restore immune function through the activation of $\mathrm{T}$-cells and $\mathrm{T}$-cell-mediated immune responses against tumor cells. PD-1, a transmembrane protein in the immunoglobulin superfamily (IgSF) expressed on T-cells, functions as an immune checkpoint that negatively regulates T-cell activation and effector function when activated by its ligands prog rammed cell death-1 ligand 1 (PD-L1; cluster of differentiation 274; CD274) or 2 (PD-L2); it plays an important role in tumor evasion from host immunity. 